\title{
Prediction of Autism Spectrum Disorder Using Rough Set Theory
}

\author{
V.Geetha ${ }^{1}$ and V. Jalaja Jayalakshmi ${ }^{1}$ \\ ${ }^{1}$ Department of Computer Applications, Kumaraguru \\ College of Technology, Coimbatore, India
}

\section{ABSTRACT}

Autism Spectrum Disorder (ASD) is a neurological disease that starts early in childhood and persists throughout a person's life. It is a condition linked with brain development and influences a person's behaviour and their interaction with others. Autism has a wide range of symptoms which can vary from person to person. There is no direct medical test to diagnose ASD disorder and hence trained physicians are needed to oversee the person's behaviour development to detect it. There is no cure for ASD, and early detection of the illness will be able to make significant quality improvements in the behaviour of the affected person. Machine Learning techniques are widely used to identify the factors associated with the disease, thus helping in early detection. This paper attempts to explore the possibilities of analyzing the autism data sets of adults using rough set theory and predict the main factors associated with the disorder for providing an early treatment. A comparative performance analysis of the results is done using two rough set algorithms, and the results indicate that the genetic algorithm gives a better performance in this domain.

KEY WORDS: AUTISM SPECTRUM DISORDER, MACHINE LEARNING, RECEIVER OPERATING CHARACTERISTICS (ROC) CURVE, REDUCTS, ROUGH SETS.

\section{INTRODUCTION}

Autism is a brain developmental disorder that starts early in childhood and affects the person's behavior amongst several age groups. It has a wide range of symptoms that varies from person to person. The prevalence of autism is increasing at an alarming rate and has mainly been found to occur in boys more than girls. Research shows that currently, autism in boys occurs at a $4: 1$ ratio and in girls, a 7:1 ratio. The overall increase in worldwide autism reporting can be attributed to a general rise in awareness of the disorder, improving technology that can

\section{ARTICLE INFORMATION}

*Corresponding Author: geetha.v.mca@kct.ac.in

Received 15th Oct 2020 Accepted after revision 4th Dec 2020

Print ISSN: 0974-6455 Online ISSN: 2321-4007 CODEN: BBRCBA

Thomson Reuters ISI Web of Science Clarivate Analytics USA and Crossref Indexed Journal

\section{Clarivate
Analytics}

NAAS Journal Score 2020 (4.31) SJIF: 2020 (7.728)

A Society of Science and Nature Publication,

Bhopal India 2020. All rights reserved.

Online Contents Available at: http//www.bbrc.in/

Doi: http://dx.doi.org/10.21786/bbrc/13.11/21 diagnose the disease, and overall detectability measures that have allowed healthcare professionals to identify the condition at an earlier age. However, it is also plausible to say that unknown risk factors that occur in day-today life contribute to the rise in autism cases around the world and, to prove this point, further research is needed to elaborate on this stance. Data mining is the method of finding patterns from vast amounts of data stored in large repositories. (Han and Kamber, 2001). These methods can handle and process large-scale information (Reidmiller,1994). The modeling techniques of data mining include classification, clustering, regression, sequential patterns etc.

Classification assigns objects to a predefined class and is an essential task of data mining. A single classification algorithm will not be able to provide good results in all cases. The choice of an optimal classification algorithm highly depends on the problem domain. Data mining has great potential in the health care industry and is becoming more and more popular. Medical databases store a lot of information about the patients and their

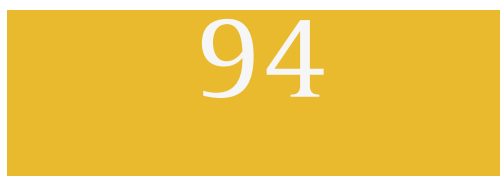


medical conditions. Classification of disease disorders from these medical databases is very challenging with standard data mining algorithms because knowledge is incomplete (Hassanien and Ali, 2004). Various algorithms are used to classify the autism data, and the authors have concluded that Multilayer perceptron algorithm gives the highest classification accuracy (Jalaja et al,. 2019).

The use of Rough set methodology in the data mining field has increased. Rough set theory was initially proposed to address vagueness in classifying the objects in each set (Pawlak, 1998). It is based on the principle that every object has some information associated with it, and objects having same information are similar and can be categorized to the same class. Rough set applications are widely used today in the medical, educational fields and other industrial applications. Rough sets are applied for bioinformatics related data (Cao et al., 2006) and to biomedical datasets (Revett, 2005) and have generated a highly accurate classifier. A rule-based disease identification system from ECG has been created (Mitra et al. 2006). The behavior of decision trees and rough set algorithms have been examined (Perzyk and Soroczynski, 2010) in industrial applications, and it has been found out that rough set algorithms can be used when a detailed set of rules are required. Rough sets have been compared with decision trees in several applications (Daubie et al., 2002, Mak and Munakata, 2002). Rough set algorithms are used to classify students' performance in adaptive E-assessment (Nandakumar et al., 2014).

Since autism has no known cure, behavioral studies are the primary way to detect autism currently; further research on these lines would prove to be beneficial to detect and possibly slow down the progression of this developmental disorder. The aim of this paper is to use rough set theory on the UCI Autism adult data set for forecasting autistic symptoms at an early stage. The organization of the paper is as follows: the theoretical aspects of the rough set algorithms are discussed in Section 2. Section 3 discusses experimental setup, results, and analysis. Future work is discussed in Section 4.

\section{MATERIAL AND METHODS}

Rough Set Theory: A Rough set is a mathematical method to analyze data stored in tabular form (Dubois and Prade 1991). Rough Set Theory finds equivalence classes from the training data. The objects that form the equivalence class are indiscernible, i.e. are identical about the attributes that explains the data.

Johnson's Algorithm: Johnson's algorithm follows a greedy strategy using heuristics for generating the reducts. It begins with an empty set $\mathrm{S}$ and counts the occurrence of each of the attributes within the discernibility clause in each iteration. The attribute that has the maximum count ' $\mathrm{f}$ ' is added into $\mathrm{S}$ and is removed from the clause. The algorithm continues till all the attributes in the clause are removed from the discernibility function, and it returns $\mathrm{S}$, which contains the reduct. This algorithm will generate a single reduct with a minimum number of attributes and does not support approximate solutions.

Genetic algorithm: Genetic Algorithm (GA) is based on heuristic approach and is inspired by organism evolution. It generates a large set of potential solutions to a problem. It evaluates the obtained solution using a fitness function. These results produce new solutions. The parent solutions with improved fitness solutions have better chances of reproduction. The reducts generated using GA begins with initializing the chromosomes from the rough set discernibility clause. The three basic processes of the genetic algorithm are generating an initial population, fitness function evaluation and reproduction.

\section{RESULTS AND DISCUSSION}

Experimental Setup: The proposed work was carried out using an open-source data set," Autism Adult data set” from the UCI Machine Learning Repository (Dua D and Graff C, 2019), which had 704 observations with 21 variables. The data set was analyzed using rough set theory. The results obtained using different algorithms in rough set theory were compared using various prediction measures. Rosetta is a toolkit application using the rough set methodology used for analyzing data stored in the form of tables.

A framework for rough set-based classification process: A rough set-based model has been developed on autism adult data set for finding decision rules to predict the occurrence of Autism Spectrum Disorder using Rosetta rough set tool. The steps in the application of rough set process are shown in Figure 1.

Figure 1: A Framework For Rough Set-Based Classification Process

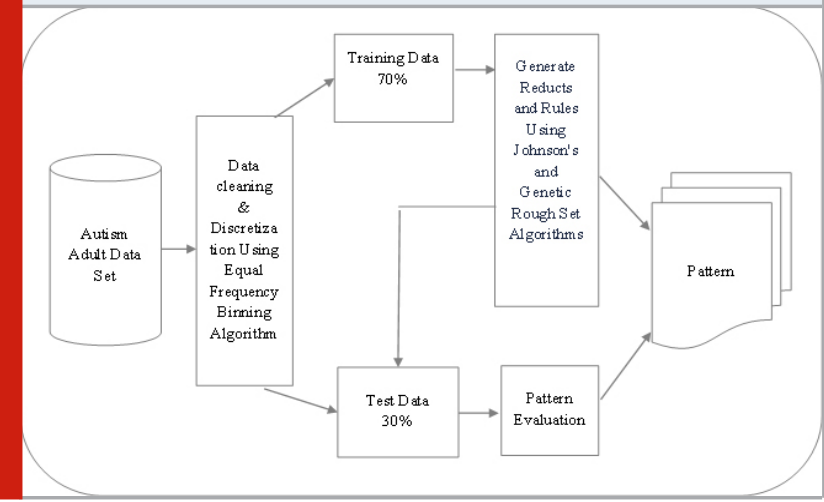

Data Preprocessing: The data set has 21 variables, out of which only two variables (age and result) are numeric, and the remaining variables are categorical/binary. There are 704 observations, out of which 189 has been classified as 'having ASD' and the remaining 515 as 'not having ASD'. It has been observed that there is a bias towards the number of observations not having ASD. The attributes 'age-desc' and 'used-app before' has the same value for all the observations and will not contribute much towards decision making. Hence, they 
are removed from the autism data set using the Rosetta tool. The data set has 15 attributes and 1 class variable after the cleaning step.

In data discretization, each continuous variable is converted into an interval-based value. Rosetta tool has a few discretization algorithms, the numerical attribute age is discretized using equal frequency binning algorithm and is shown in Figure 2. After discretization, the database is arbitrarily divided into two different datasets namely training and test data sets. A model is built using the training set and is then evaluated on the test set. Different sample sizes are randomly selected from the data set for various split factors (seed) ranging from 0.1 to 0.9 and are used for training and testing purposes.

Figure 2: Data Discretization By Equal Frequency Binning Algorithm

\begin{tabular}{|c|c|c|c|c|c|}
\hline age & gender & ethnicity & Jaundice & $\begin{array}{c}\text { contry_of_r } \\
\text { es }\end{array}$ & ASD \\
\hline$[24,32)$ & $\mathrm{f}$ & White-Europ & no & United State & NO \\
\hline$[24,32)$ & $\mathrm{m}$ & Latino & no & Brazil & NO \\
\hline$[24,32)$ & $\mathrm{m}$ & Latino & yes & Spain & YES \\
\hline$\left[32,{ }^{*}\right)$ & $\mathrm{f}$ & White-Europ & no & United State & NO \\
\hline$\left[32,{ }^{*}\right)$ & $\mathrm{m}$ & Others & yes & "United State & YES \\
\hline$\left[{ }^{*}, 24\right)$ & $\mathrm{f}$ & Black & no & "United State & NO \\
\hline$\left[32,{ }^{*}\right)$ & $\mathrm{m}$ & White-Europ & no & "New Zealan & NO \\
\hline$[24,32)$ & $\mathrm{m}$ & White-Europ & no & "United State & NO \\
\hline$\left[{ }^{*}, 24\right)$ & $\mathrm{m}$ & Asian & yes & Bahamas & YES \\
\hline$\left[32,{ }^{*}\right)$ & $\mathrm{m}$ & White-Europ & no & United State & YES \\
\hline$\left[{ }^{*}, 24\right)$ & $\mathrm{f}$ & "Middle Easte & no & Burundi & NO \\
\hline$\left[{ }^{*}, 24\right)$ & $\mathrm{m}$ & "Middle Easte & no & "New Zealan & NO \\
\hline$[24,32)$ & $\mathrm{m}$ & "Middle Easte & no & Jordan & NO \\
\hline$[24,32)$ & $\mathrm{m}$ & White-Europ & no & Ireland & NO \\
\hline$\left[32,{ }^{*}\right)$ & $\mathrm{f}$ & "Middle Easte & no & "United Arab & NO \\
\hline \hline
\end{tabular}

Figure 3: Few Reducts Generated Using Johnson's Algorithm

T. Johnson 40

\begin{tabular}{|c|c|c|}
\hline & Reduct & Support Length \\
\hline
\end{tabular}

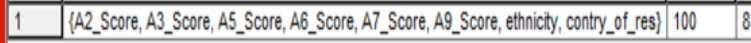

Figure 4: Few Reducts Generated Using Genetic Algorithm

\begin{tabular}{|c|c|c|c|}
\hline & Reduct & Support & Lengtt \\
\hline 2 & 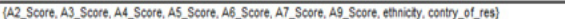 & 100 & \\
\hline 3 & 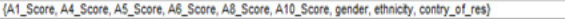 & 100 & 9 \\
\hline 4 & 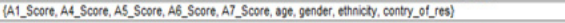 & 100 & 9 \\
\hline 5 & 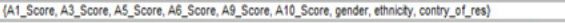 & 100 & \\
\hline 6 & 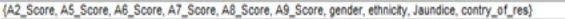 & 100 & \\
\hline 7 & 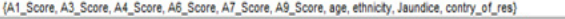 & 100 & 10 \\
\hline 8 & 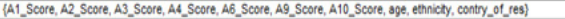 & 100 & 10 \\
\hline 9 & 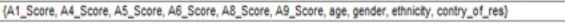 & 100 & 10 \\
\hline 10 & 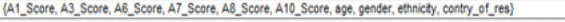 & 100 & 10 \\
\hline & 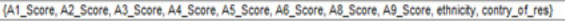 & 100 & 10 \\
\hline 12 & [A2_Score, A3__score, A4_Score, A6__Score, A7__Score, AB__Score, A9__Score, A10_Score, age, contry_of_rese) & 100 & 10 \\
\hline 13 & 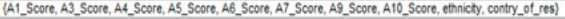 & 100 & 10 \\
\hline 14 & 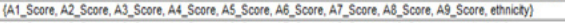 & 100 & 10 \\
\hline 15 & 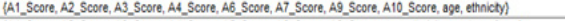 & 100 & 10 \\
\hline 16 & 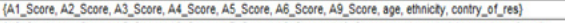 & 100 & 10 \\
\hline 17 & 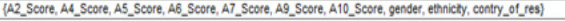 & 100 & 10 \\
\hline 18 & 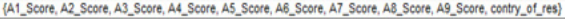 & 100 & 10 \\
\hline 19 & 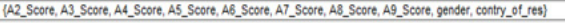 & 100 & 10 \\
\hline 20 & 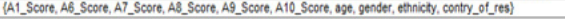 & 100 & 10 \\
\hline 21 & 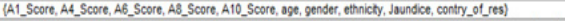 & 100 & 10 \\
\hline & 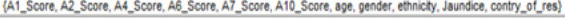 & 100 & 11 \\
\hline
\end{tabular}

Generation of reducts: In rough set theory, attribute reduction is achieved through generation of reducts, which is an essential feature in the rough set application to data mining. A reduct of an attribute set describes a minimum set of attributes representing the various classes of the data set. The objective of rough set theory is to derive minimal decision rule using reducts. The 96 reducts are then used on the autism data set to derive the decision rules. These rules can then be used to categorize new instances of data and predict the unknown class label. The Rosetta tool has various algorithms based on heuristics for the formation of reducts. In this study, attribute reduction and classification are carried out using Johnson's algorithm and Genetic algorithm. The reduct generated by the algorithms for seed value of 4 are shown in Figures 3 and 4, respectively.

Generation of Decision Rules: Rough set methods generate rules which are used for predicting the output. The reduct algorithms in the Rosetta tool also generate IF_THEN rules consisting of predictor variables and a decision descriptor variable, which can be used for classification. A sample of the rules generated by Johnson's algorithm is shown in Figure 5. To determine the strength of the classifiers, they are validated on the training set and the final model is tested on the test set. Both Johnson's and genetic algorithms are applied to the training sets resulting from different training and test split. Figure 6 shows the performance of these algorithms for seed values ranging from 0.1 to 0.9 .

Figure 5: Rules Created By The Johnson's Algorithm (Sample)

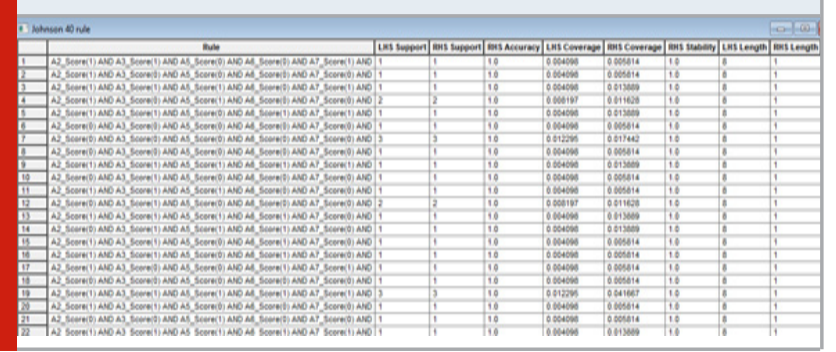

Figure 6: Performance of the Algorithms On The Autistic Data Set

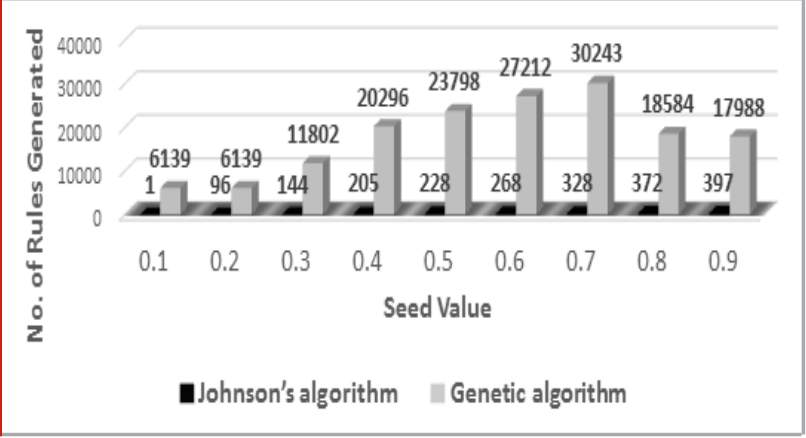

The rules contain 15 predictor variables that predict the outcome variable 'ASD'. It can be seen from Figure 6 , the total rules generated by Johnson's, and genetic algorithms were 144 and 11802 respectively. The total rules generated by the genetic algorithm is more than that of Johnson's algorithm for the various sample sizes. This is because GA generated a greater number of reducts.

\section{Evaluation of the Proposed Model}

Classification accuracy: The performance of the classifiers 
is measured using accuracy, which is displayed using confusion matrix (Witten et al. 2011). The confusion matrix shows the number of correct and incorrect predictions given by the rules on the test data. In the binary classification, the objects in the test set will belong either to the positive decision class (Yes) or to the negative decision class (No). The confusion matrix generated by the Rosetta tool for genetic algorithm with seed value six is depicted in Figure 7.

Figure 7: Confusion Matrix For 40\% Test Samples Using Genetic Algorithm

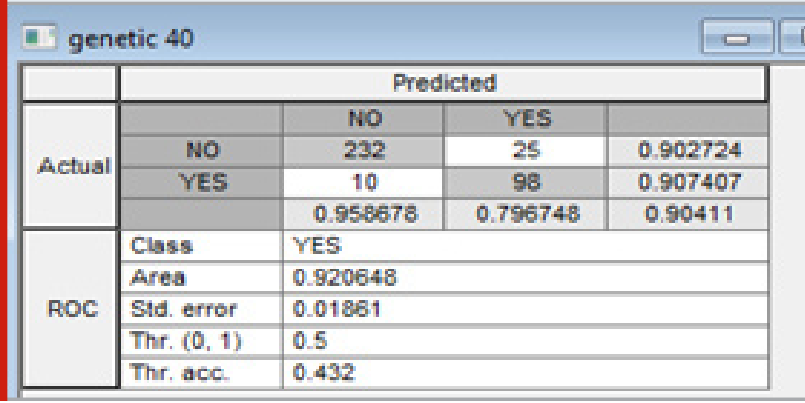

Table 1. Classification Accuracy \% of Rough Set Algorithms

\begin{tabular}{|l|c|c|}
\hline $\begin{array}{l}\text { Seed } \\
\text { Value }\end{array}$ & $\begin{array}{l}\text { Johnson's } \\
\text { algorithm }\end{array}$ & $\begin{array}{c}\text { Genetic } \\
\text { algorithm }\end{array}$ \\
\hline 0.1 & 0.047 & 0.803 \\
\hline 0.2 & 0.549 & 0.893 \\
\hline 0.3 & 0.585 & 0.874 \\
\hline 0.4 & 0.594 & 0.865 \\
\hline 0.5 & 0.595 & 0.868 \\
\hline 0.6 & 0.518 & 0.904 \\
\hline 0.7 & 0.589 & 0.899 \\
\hline 0.8 & 0.507 & 0.854 \\
\hline 0.9 & 0.332 & 0.900 \\
\hline
\end{tabular}

Figure 8: Auc Comparison Between The Classifiers

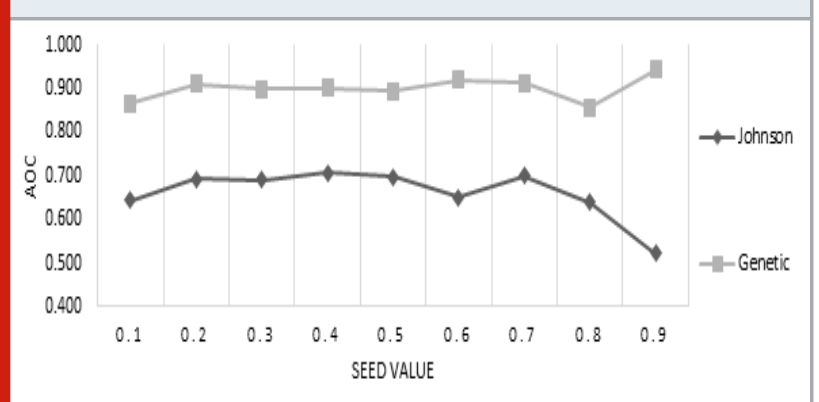

It can be seen that the accuracy for classifying $40 \%$ test samples using the genetic algorithm is $90.4 \%$. Out of 365 samples in the test data set, 330 samples were classified correctly, and only a few samples were not classified correctly. The accuracy percentage of the two algorithms are given in Table 1. It is seen that the genetic algorithm has the highest accuracy among the two algorithms for all the seed values.

Receiver Operating Characteristic (ROC) Curves: Receiver Operating Characteristic (ROC) curves are helpful for evaluating the accuracy of predicted values (Fawcett 2004). It is a graphical representation for assessing the predicted and actual values in a classifier model. It is used to measure the performance of a test. ROC curves examine the relationship between sensitivity and specificity. The Area Under Curve (AUC) provides a measure of bias that how well a classifier can distinguish objects in class a label. AUC is normally recognized as the best measure for assessing the performance of classifiers (Øhrn 1999). The AUC values obtained for both the algorithms are shown in Figure 8. It is seen from the above Figure 8 that the genetic algorithm has greater AUC value is for all the seed values and it outperforms Johnson's algorithm. The dataset sizes that result in prediction models with the largest area under their ROC curves is selected as the better classification method for autism data.

\section{CONCLUSION AND FUTURE WORK}

A rough set model using ROSETTA tool kit has been applied on the UCI autism adult data set for predicting the presence or absence of ASD. Johnson's and genetic algorithms were used for classification task and the results reveal that the genetic algorithm has a superior performance over Johnson's algorithm with classification accuracy and ROC curve measures.

\section{REFERENCES}

Cao Y Liu S Zhang L Qin J Wang J and Tang K (2006) Prediction of Protein Structural Class with Rough Sets, BMC Bioinformatics, pp.7-20.

Daubie M Levecq P and Meskens N (2002) A Comparison of Rough Sets and Recursive Partitioning Induction Approaches: An Application to Commercial Loans, International Transactions in Operational Research, pp.681-694.

Dua D and Graff C (2019) UCI Machine Learning Repository [http://archive.ics.uci.edu/ml]. Irvine, CA: University of California, School of Information and Computer Science.

Dubois D and Prade H (1991) Foreword In: Rough Sets - Theoretical Aspects of Reasoning about Data, by Z. Pawlak. Kluwer, Dordrecht, Netherlands.

Fawcett T (2004) ROC Graphs: Notes and Practical Considerations for Researchers, Kluwer Academic Publishers.

Han J and Kamber M (2001) Data Mining Concepts and Techniques, Morgan Kaufmann Publishers, ISBN 1-55860-489-8

Hassanien AE and Ali JMH (2004) Rough Set Approach for Generation of Classification Rules of Breast Cancer Data, INFORMATICA, Vol.15, No.1, pp.23-38.

Jalaja Jayalakshmi V Geetha V and Vivek R (2019) Classification of Autism Spectrum Disorder Data using Machine Learning Techniques, International Journal of 
Engineering and Advanced Technology (IJEAT), ISSN: 2249 - 8958, Volume-8 Issue-6S, pp. 565-569.

Mak B and Munakata T (2002) Rule Extraction from Expert Heuristics: A Comparative Study of Rough Sets with Neural Networks and ID3, European Journal of Operational Research, Vol.136, pp.212-229.

Mitra S Mitra M. and Chaudhuri BB (2006) An Approach to Rough Set Based Disease Inference Engine for ECG Classification”, Rough Sets and Current Trends in Computing, Proceedings of 5th International Conference, RSCRC, pp.400-406

Nandakumar GS Geetha V Surendiran B and Thangasamy S (2014) A Rough Set Based Classification Model for Grading in Adaptive E-Assessment, International Reviews on Computers \& Software, Vol.9, No.7, pp.1169 -1177 .

Øhrn A (1999) Discernibility and Rough Sets in Medicine: Tools and Applications, PhD Thesis, Norwegian University of Science and Technology,
Trondheim, Norway.

Pawlak Z (1998) Reasoning about Data - A Rough Set Perspective, RSCTC'98, Lecture Notes in Artificial Intelligence, Vol.1424, pp.25-34.

Perzyk M. and Soroczynski A (2010) Comparative Study of Decision Trees and Rough Sets Theory as Knowledge Extraction Tools for Design and Control of Industrial Processes, World Academy of Science, Engineering and Technology, pp.84- 90.

Reidmiller M (1994) Advanced Supervised Learning in Multi-layer Perceptions from Back Propagation to Adaptive Learning Algorithms, Computer Standards Interfaces, pp.265-278.

Revett K (2005) A Rough Set Based Classifier for Primary Biliary Cirrhosis, International Conference on Computers as a Tool, pp.1128-1131.

Witten H Frank E and Hall MA (2011) Data Mining: Practical Machine Learning Tools and Techniques, Morgan Kaufmann Publishers. 